\title{
QUALIDADE DO SONO E TOLERÂNCIA AO ESFORÇO EM PORTADORES DE APNEIA OBSTRUTIVA DO SONO
}

\author{
SLEEP QUALITY AND STRESS TOLERANCE IN PATIENTS WITH OBSTRUCTIVE SLEEP APNEA
}

CALIDAD DEL SUEÑO Y LA TOLERANCIA AL ESTRÉS EN PACIENTES CON APNEA

OBSTRUCTIVA DEL SUEÑO

Aliny Priscilla do Nascimento ${ }^{1}$ (Bióloga)

Vívian Maria Moraes Passos ${ }^{2}$ (Fisioterapeuta)

Rodrigo Pinto Pedrosa ${ }^{3}$ (Médico Pneumologista)

Maria do Socorro BrasileiroSantos 4 (Educadora Física)

Isly Maria Lucena de Barros ${ }^{3}$ (Médico)

Laura Olinda Bregieiro Fernandes Costa ${ }^{3}$ (Tocoginecologista)

Amilton da Cruz Santos ${ }^{4}$

(Educador Físico)

Anna Myrna Jaguaribe de Lima' (Fisioterapeuta)

1. Universidade Federal Rural de Pernambuco (UFRPE), Recife, PE, Brasil.

2. Universidade Federal de Pernambuco (UFPE) Recife, PE, Brasil.

3. Universidade de Pernambuco (UPE) Recife, PE, Brasil.

4. Universidade Federal da Paraíba (UFPB) João Pessoa, PB, Brasil.

\section{Correspondência:}

Rua Olavo Bilac, 76A, 54220-060, Curado Dois, Jaboatão dos Guararapes, PE, Brasil. aliny_biologia7@yahoo.com.br

\section{RESUMO}

Introdução: O sono é um estado natural e recorrente, no qual acontecem processos neurobiológicos importantes. A má qualidade do sono está diretamente associada com piores indicadores de saúde. A qualidade do sono pode ser medida objetiva e subjetivamente por métodos como a polissonografia, que é o padrão de referência, ou por meio de testes e questionários, como o índice de qualidade de sono de Pittsburgh (IQSP). Objetivo: Correlacionar a qualidade do sono com a tolerância ao esforço em pacientes portadores da síndrome da apneia/hipopneia obstrutiva do sono (SAHOS). Métodos: Participaram do estudo 63 indivíduos (57 mulheres e seis homens), média de idade de 51,7 \pm 6,6 anos; índice de massa corpórea (IMC) média de 28,2 $\pm 5,0 \mathrm{~kg} / \mathrm{m}^{2}$ ); índice de apneia/hipopneia (IAH) médio de 7,3 \pm 10,50 eventos/hora, verificado através da polissonografia. Para a avaliação da qualidade do sono, os participantes responderam ao IQSP, e para a avaliação da tolerância ao esforço, realizaram o teste de caminhada de 6 minutos (TC6M). Resultados: Não houve correlação entre o IQSP e o TC6M $\left(R_{s}=-0,103620, p=0,419\right)$, assim como entre o IAH e o TC6M $\left(R_{s}=-0,000984, p=0,9939\right)$. Podemos sugerir que a qualidade do sono e a gravidade da SAHOS não afetam a tolerância ao esforço dos indivíduos com SAHOS. Conclusão: Estudos com uma amostra maior, levando-se em consideração a estratificação pela gravidade da SAHOS e utilizando métodos mais acurados de avaliação da capacidade funcional, devem ser realizados, a fim de que resultados mais abrangentes possam ser obtidos.

Palavras-chave: apneia, tolerância ao exercício, transtornos do sono, sono, exercício.

\begin{abstract}
Introduction: Sleep is a natural and recurring state, in which important neurobiological processes take place. Poor quality of sleep is directly associated with worse health indicators. Sleep quality can be measured objectively and subjectively by methods such as polysomnography, which is the gold standard, or tests and questionnaires as the Sleep Quality Index in Pittsburgh (PSQI). Objective: To correlate the quality of sleep with exercise tolerance in patients with obstructive sleep apnea-hypopnea syndrome (OSAHS). Methods: The study included 63 subjects ( 57 women and 6 men) aged $51.7 \pm 6.6$ years, with body mass index (BMI) $28.2 \pm 5.0 \mathrm{~kg} / \mathrm{m} 2$, apnea/hypopnea index (AHI) 7.3 $\pm 10: 50$ events/hour, assessed by polysomnography. To assess the quality of sleep, participants responded the PSQI instrument and were submitted to the exercise tolerance test through the 6-minute walk test (6MWT). Results: There was no correlation between the PSQI and the 6MWT (Rs $=-0.103620, p=0.419$ ), and between the 6MWT and the AHI $(R=-0,000984, p=0.9939)$. According to the results of this work, we suggest that the quality of sleep and the severity of OSAHS did not affect the exercise tolerance of individuals with OSAHS. Conclusion: Studies with a larger sample, taking into account the stratification by severity of OSAHS and using more accurate methods of functional capacity evaluation should be conducted, so that more comprehensive results can be obtained.
\end{abstract}

Keywords: apnea, exercise tolerance, sleep disorders, sleep, exercise.

\section{RESUMEN}

Introducción: El sueño es un estado natural y recurrente durante el cual acontecen procesos neurobiológicos importantes. La mala calidad del sueño está asociada directamente con peores indicadores de salud. La calidad del sueño puede ser medida objetiva y subjetivamente mediante métodos como la polisomnografía, que es el estándar de referencia, o pruebas y cuestionarios como el índice de calidad de sueño de Pittsburgh (ICSP), Objetivo: Correlacionar la calidad del sueño con la tolerancia al esfuerzo en pacientes portadores del síndrome de la apneal hipopnea obstructiva del sueño (SAHOS). Métodos: Participaron en el estudio 63 individuos (57 mujeres y seis hombres), edad promedio de 51,7 \pm 6,6 años; índice de masa corpórea (IMC) 28,2 \pm $5,0 \mathrm{~kg} / \mathrm{m}^{2}$ ); índice de apnea/hipopnea (IAH) 7,3 $\pm 10,50$ eventos/hora, verificado por medio de la polisomnografía. Para la evaluación de la calidad del sueño, los participantes respondieron al ICSP y para la evaluación de la tolerancia al esfuerzo, realizaron la prueba de caminata de 6 minutos (PC6M). Resultados: 
No hubo correlación entre el ICSP y la PC6M $\left(R_{s}=-0,103620, p=0,419\right)$, ni entre el IAH y la PC6M $\left(R_{s}=-0\right.$, $000984, p=0,9939$ ). Podemos sugerir que la calidad del sueño y la gravedad del SAHOS no afectan a la tolerancia al esfuerzo de los individuos con SAHOS. Conclusión: Se deben realizar los estudios con una muestra mayor, teniéndose en consideración la estratificación según la gravedad del SAHOS y utilizándose métodos más precisos para evaluación de la capacidad funcional, a fin de que se puedan obtener resultados de más alcance.

Palabras clave: apnea, la tolerancia al ejercicio, los trastornos del sueño, sueño, ejercicio.

\section{INTRODUÇÃO}

O sono é um estado natural e recorrente de desligamento e de irresponsividade do organismo ao ambiente, durante o qual acontecem processos neurobiológicos que mantêm a integridade física e cognitiva do organismo ${ }^{1}$. A má qualidade do sono está diretamente associada a pior saúde, aumento da utilização dos serviços de saúde, do absenteísmo do trabalho e do risco para transtornos psicológicos, incluindo depressão². Uma noite de sono mal dormida causa vários problemas, como a sonolência diurna excessiva, podendo tornar-se um problema de saúde pública, aumentando o risco de mortalidade como, por exemplo, em acidentes automobilísticos ${ }^{3}$.

Para a verificação adequada das propriedades quantitativas e qualitativas do sono, podem-se utilizar tanto medidas objetivas quanto subjetivas. Entre as medidas objetivas, a polissonografia é o padrão-ouro que reconhece tanto o sono normal quanto o alterado ${ }^{4}$. Dentre as medidas subjetivas, existem os testes e questionários de qualidade do sono, como o índice de qualidade de sono Pittsburgh (IQSP) ${ }^{5}$, que tem uma sensibilidade de $89,6 \%$ e especificidade de $86,5 \%$ para a identificação de casos com distúrbio do sono ${ }^{6}$.

Esta qualidade do sono pode ser afetada por distúrbios específicos. Um distúrbio muito frequente durante o sono é a síndrome da apneia/hipopneia obstrutiva do sono (SAHOS), caracterizada por interrupção total (apneia) e/ou parcial (hipopneia) do fluxo de ar nas vias aéreas superiores por, no mínimo dez segundos, decorrente do colapso inspiratório dessas vias ${ }^{7}$. Os efeitos agudos e repetitivos de apneias e hipopneias podem contribuir para doenças cardiovasculares que frequentemente coexistem com a síndrome ${ }^{8}$. Estima-se que aproximadamente $5 \%$ dos indivíduos adultos de países ocidentais tem a SAHOS 9 .

De acordo com ROUX et al. ${ }^{10}$, pacientes com SAHOS têm maior predisposição à hipertensão, arritmias, insuficiência cardíaca, infarto, acidente vascular encefálico e possível morte súbita durante o sono. A SAHOS pode funcionar como um gatilho de ativação simpática persistente, contribuindo com a elevação da pressão arterial e com alterações de ritmo cardíaco ${ }^{11}$. Tais consequências sugerem que a SAHOS interfere de maneira sistêmica no organismo podendo afetar, inclusive, a capacidade funcional do indivíduo.

Uma forma de avaliar esta capacidade funcional de maneira prática e submáxima é o teste de caminhada de 6 minutos (TC6M) que avalia a resposta global e integrada de todos os sistemas envolvidos durante o exercício. Entretanto, ele não fornece informações específicas sobre a função de cada um dos diferentes órgãos e sistemas envolvidos no exercício ou o mecanismo de limitação ao exercício, como é possível com um teste cardiopulmonar máximo. Porém, a maioria das atividades diárias é desenvolvida em níveis submáximos de esforço. Portanto, o TC6M pode refletir melhor o nível de exercício funcional para atividades físicas diárias ${ }^{12}$. Desta forma, o objetivo do presente trabalho foi correlacionar a qualidade do sono com a tolerância ao esforço em pacientes portadores de SAHOS.

\section{MÉTODOS}

A amostra foi composta por 63 indivíduos (57 mulheres e seis homens) que faziam parte do banco de dados do laboratório do sono e do coração do Pronto-Socorro Cardiológico de PernambuCo (PROCAPE) no período de julho a novembro de 2011 e que se enquadravam nos seguintes critérios de inclusão: indivíduos não obesos, com sobrepeso ou obesos de até grau $\|\left(18 \mathrm{Kg} / \mathrm{m}^{2}<\mathrm{IMC} \leq\right.$ $40 \mathrm{Kg} / \mathrm{m}^{2}$ ); idade entre 30 e 60 anos, e capacidade de realização dos exames propostos. Foram considerados critérios de exclusão: obesos mórbidos (IMC $\geq 40 \mathrm{Kg} / \mathrm{m}^{2}$ ); outros distúrbios respiratórios que não a SAHOS; tabagistas; instabilidade ou desconforto durante qualquer um dos exames. Após a seleção, os indivíduos foram divididos em dois grupos: G1, indivíduos não portadores de SAHOS ( $n=39$ ) e $G 2$, indivíduos portadores de SAHOS $(n=24)$. Os indivíduos convidados a participar da pesquisa eram informados dos objetivos e assinaram o termo de consentimento de pesquisas envolvendo seres humanos segundo as Diretrizes e Normas Regulamentadoras de Pesquisas Envolvendo Seres Humanos (Resolução No 196, de 10 de outubro de 1996) do Conselho Nacional de Saúde. A pesquisa foi aprovada pelo Comitê de Ética em Pesquisa para Seres Humanos da Universidade Federal de Pernambuco (CAAE - No 0367.0.172.000-10).

Foram mensurados o peso $(\mathrm{kg})$ e a altura $(\mathrm{m})$ corporal, utilizando-se uma balança antropométrica, e o índice de massa corpórea - IMC - $\left(\mathrm{kg} / \mathrm{m}^{2}\right)$ foi calculado dividindo-se o valor do peso (kg) pela altura ${ }^{2}(\mathrm{~m})$.

Os pacientes foram submetidos à polissonografia de noite inteira. Utilizando-se um sistema de polissonografia computadorizada (Healthdyne Technologies, Respironics, Alice $3^{\circledR}$ - EUA) do Laboratório do Sono e do Coração do PROCAPE. Os parâmetros mensurados foram eletrocardiograma (ECG), pressão arterial (PA), movimentos torácicos e abdominais (pletismografia), saturação periférica de oxigênio $\left(\mathrm{SpO}_{2}\right)$ (oximetria de pulso), fluxo aéreo nasal/bucal (termistores), posição corporal e ronco (microfone). Foram calculados os números de apneias e hipopneias, para obter o índice de apneia/hipopneia (IAH) estes eventos respiratórios foram classificados usando critérios da American Academy of Sleep Medicine ${ }^{13}$.

O questionário índice de qualidade do sono de Pittsburgh (IQSP) foi respondido na sala do Laboratório do Sono e do Coração do PROCAPE. O IQSP consiste em um questionário de avaliação subjetiva da qualidade do sono de um indivíduo com relação ao mês anterior.

O questionário contém dez perguntas que são combinadas em sete pontuações com componentes clinicamente derivados em informações quantitativas e qualitativas sobre o sono do indivíduo, cada uma com o mesmo peso de zero a três. Os sete componentes da escala são adicionados para que se obtenha uma pontuação global que varia de 0 a 21, com a escala mais alta indicando pior qualidade do sono ${ }^{14}$.

Teste de Caminhada de 6 minutos: 0 teste de caminhada de seis minutos (TC6M) foi realizado em um corredor de 54 metros de 
comprimento, de superfície plana e sólida, que os pacientes percorreram a pé, no quarto andar do PROCAPE, onde a sala do laboratório do sono está localizada. O objetivo do TC6M foi mensurar o maior número de voltas possíveis, no seu ritmo normal de caminhada de cada paciente durante seis minutos. O TC6M reflete o nível de exercício funcional para atividades físicas diárias ${ }^{12}$.

\section{Análise Estatística}

Para examinar a normalidade da distribuição dos dados foi realizado o teste de Kolmogorov-Smirnov. Para verificar a diferença intergrupos foi realizado o teste de Mann-Whitney. Para o estabelecimento das correlações utilizou-se o coeficiente de Spearman. Os valores foram apresentados como média \pm desvio padrão. $O$ valor de $p<0.05$ foi considerado como nível de significância estatística. Para os cálculos foi utilizado o programa Statistica ${ }^{\circledR} 7.0$ para Windows ${ }^{\circledR}$.

\section{RESULTADOS}

A tabela 1 mostra os dados referentes às características da amostra. Não houve diferenças na idade e na distância percorrida no teste de caminhada de 6 minutos (TC6M), quando comparamos os dois grupos. Os pacientes do G2 apresentaram maior peso $(77,7 \pm 13,2 \mathrm{~kg})$, altura $(1,59 \pm 0,06 \mathrm{~m}), \operatorname{IMC}\left(15,7 \pm 13,3 \mathrm{~kg} / \mathrm{m}^{2}\right)$ e IQSP $(9,9 \pm 3,5)$ quando comparados ao G1. Foram observadas comorbidades, tais como: hipertensão arterial sistêmica e dislipidemia. Dos 63 indivíduos do estudo, 45 apresentavam ao menos uma destas duas comorbidades, 14 possuíam ambas e 18 não apresentavam tais doenças.

Não houve correlação significativa entre o índice de qualidade do sono de Pittsburgh e a distância percorrida no teste de caminhada de seis minutos $\left(R_{s}=-0,103620, p=0,419\right)$. (figura 1$)$.

Da mesma forma, não foi encontrada correlação significativa entre o índice de apneia/hipopneia e a distância percorrida no teste de caminhada de seis minutos $\left(R_{s}=-0,000984, p=0,9939\right)$ (figura 2).

Quando correlacionado o IQSP e o TC6M dos pacientes portadores de SAHOS moderada e grave, observa-se uma correlação negativa moderada, porém, não significativa $\left(R_{s}=-0.442542, p=0.2002\right)$.

Tabela 1. Caracterização da amostra.

\begin{tabular}{c|c|c}
\hline & G1 & G2 \\
\hline \multirow{2}{*}{ Variável } & $\begin{array}{c}\text { (Controle) } \\
(\mathbf{n}=\mathbf{3 9})\end{array}$ & $\begin{array}{c}\text { (Portadores de SAHOS) } \\
(\mathbf{n}=\mathbf{2 4})\end{array}$ \\
\hline \multirow{2}{*}{ Gênero } & 2 homens & 4 homens \\
\cline { 2 - 3 } & 37 mulheres & 20 mulheres \\
\hline IAH (Eventos/h) & $2,2 \pm 1,4$ & $15,7 \pm 13,3^{*}$ \\
\hline Idade (anos) & $51,4 \pm 6,9$ & $52,8 \pm 5,7$ \\
\hline Peso (Kg) & $65,9 \pm 14,9$ & $77,9 \pm 13,2^{*}$ \\
\hline Altura (m) & $1,56 \pm 0,07$ & $1,59 \pm 0,06^{*}$ \\
\hline IMC (Kg/m $\left.{ }^{2}\right)$ & $26,9 \pm 5,1$ & $30,5 \pm 4,2^{*}$ \\
\hline Doenças Cardiovasculares & 15 HAS & 9 HAS \\
associadas & 4 Dislipidemia & 3 Dislipidemia \\
\hline Distância percorrida & $487,4 \pm 62,7$ & $476,4 \pm 68,4$ \\
no TC6M (m) & $8,1 \pm 2,9$ & $9,9 \pm 3,5^{*}$ \\
\hline IQSP &
\end{tabular}

IAH - índice de apneia/hipopneia; IMC - índice de massa corpórea; HAS - hipertensão arterial sistêmica; TC6M - teste de caminhada de 6 minutos; IQSP - índice de qualidade do sono de Pittsburgh. * $p<0,05$ - G1 vs. G2.

\section{DISCUSSÃO}

Uma boa qualidade do sono está diretamente associada a um período de vigília adequado, sem sonolência diurna e com maior disposição para a realização das atividades de vida diária. Porém, esta qualidade do sono pode ser afetada por distúrbios como a SAHOS, que está relacionada a outras situações clínicas de morbidade tais como insuficiência cardíaca, hipertensão arterial, arritmia e obesidade.

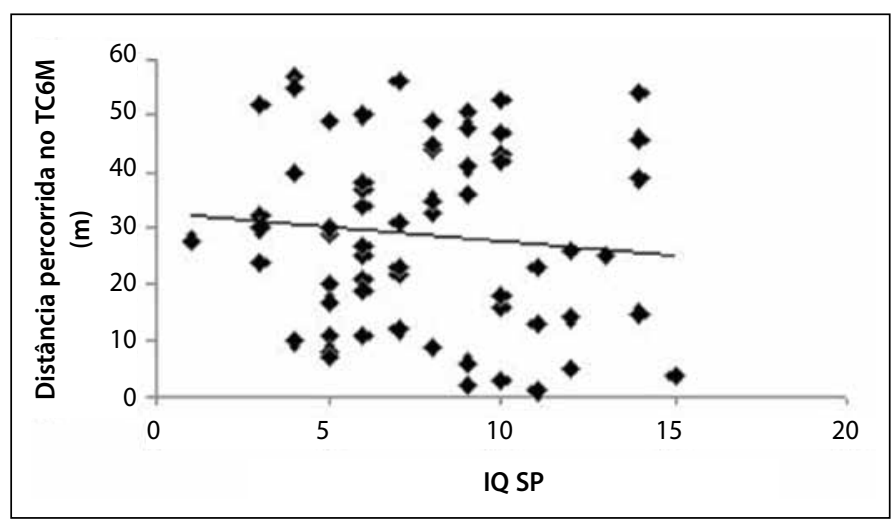

Figura 1. Correlação entre índice de qualidade do sono de Pittsburgh (IQSP) e teste de caminhada de seis minutos (TC6M). Rs $=-0,103620, p=0,419$.

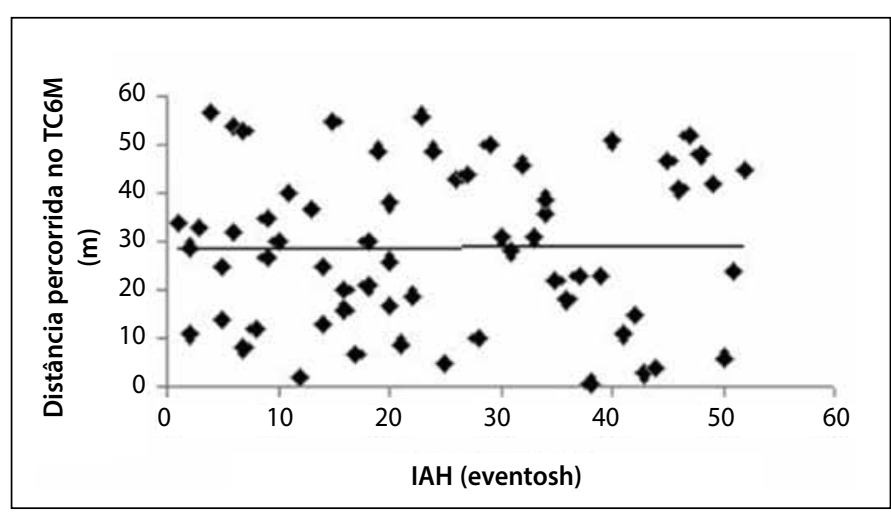

Figura 2. Correlação entre índice da apneia/hipopneia $(\mathrm{IAH})$ e teste de caminhada de seis minutos (TC6M). Rs $=-0,000984, p=0,9939$.

Na tabela 1, observamos a caracterização da amostra e a homogeneidade dos grupos estudados quanto à idade. É importante ressaltar que a ocorrência da SAHOS tem relação direta com o aumento da idade ${ }^{15}$. Quanto ao IMC, o G2 apresenta valores superiores ao G1. Entre adultos com sobrepeso, um em cada cinco possuem SAHOS leve e um em cada quinze tem essa doença de forma moderada ou grave. Em obesos, a prevalência da SAHOS chega a 40\% ${ }^{8}$. Fica claro, portanto, que a obesidade aumenta o risco de aparecimento da SAHOS, sendo a recíproca também verdadeira. Pedrosa et al. ${ }^{11}$ demonstraram que a própria apneia do sono pode predispor ao sobrepeso e à obesidade, tendo observado que 70\% dos pacientes portadores de SAHOS desenvolvem tais condições.

No que diz respeito à qualidade do sono, ao correlacionarmos os valores obtidos no IQSP com a distância percorrida no TC6M, não foi verificada correlação (Figura 1), entretanto, ambos os grupos apresentaram IQSP acima de 5 , indicando uma má qualidade do sono (Tabela 1). Contudo, quando correlacionado o IQSP com o TC6M dos pacientes com SAHOS moderada e grave, houve uma correlação moderada, porém não significativa. Possivelmente, o tamanho reduzido da amostra possa ter influenciado este resultado. Uma quantidade maior de indivíduos portadores de SAHOS e sua estratificação quanto à gravidade da doença poderia esclarecer meIhor a existência desta correlação, e se realmente a presença de SAHOS de maior gravidade afeta de maneira significativa a capacidade funcional destes pacientes. Sendo assim, o IQSP apesar de estar intimamente relacionado com medidas do sono diário, não é adequado para triar os tipos de anormalidades do sono ${ }^{16}$. Por ser uma medida altamente subjetiva, os pacientes podem não ser fidedignos na auto avaliação da sua qualidade do sono no momento de responder ao questionário.

No presente estudo, não houve correlação entre a gravidade da SAHOS e a capacidade funcional dos indivíduos (figura 2). Confirmando os nossos achados, Alameri et al. ${ }^{17}$ ao analisarem indivíduos obesos com 
e sem SAHOS e indivíduos eutróficos sem SAHOS, verificaram que, no grupo com SAHOS, os resultados TC6M não se correlacionaram com - IAH. Da mesma forma, também não houve diferença na distância percorrida quando comparados os grupos com e sem SAHOS. Apesar de não terem investigado os fatores que levaram a este resultado, os autores sugerem que fatores como força muscular, função cardíaca e fatores psicológicos, além da variação dentro da população, podem justificar esses achados. Da mesma maneira, Rizzi et al. ${ }^{18}$ observaram que pacientes não obesos com SAHOS submetidos a exercício, não apresentaram diferenças quanto ao $\mathrm{VO}_{2 \text { máx }}$ quando comparados com grupo controle de índice de massa corporal (IMC) também normal e sem a síndrome, reforçando que a SAHOS por si só, não compromete a tolerância ao esforço nos portadores da síndrome.

No entanto, Plywaczewsky et al. ${ }^{19}$ ao avaliarem a capacidade funcional através do TC6M em portadores de SAHOS, obesos, sob tratamento com CPAP, verificaram que estes indivíduos apresentavam menor tolerância ao esforço, determinada por fatores como idade, gênero (feminino), IMC e capacidade vital forçada reduzida. Da mesma maneira, no estudo realizado por Thomas et al. ${ }^{20}$, indivíduos obesos mórbidos portadores de SAHOS demonstraram diminuição do $\mathrm{VO}_{2 \text { máx }}$ em comparação com pacientes também obesos, porém sem a SAHOS, submetidos a um teste de aptidão cardiorespiratória máximo. Os autores relataram que a diminuição da capacidade funcional estaria relacionada à obesidade, que quase sempre está associada à SAHOS. Outro fato que pode ter influenciado na ausência de correlação entre IAH e capacidade funcional é a utilização de teste submáximo, que pode não ter avaliado de maneira tão acurada a capacidade funcional dos indivíduos que participaram deste estudo.

Já é comprovada a forte associação entre a SAHOS e a doença cardiovascular. Em estudo realizado por Cintra et al. ${ }^{21}$, o perfil cardiovascular de pacientes com SAHOS mais encontrado foi: obesidade, hipertensão arterial, baixos níveis plasmáticos de HDL e átrio esquerdo com diâmetro aumentado. Estes resultados corroboram com os achados de Roux et al. ${ }^{10}$, no qual pacientes com SAHOS apresentavam maior predisposição à hipertensão, arritmias, insuficiência cardíaca, infarto, acidente vascular encefálico. Sendo assim, a SAHOS e/ou essas comorbidades interferem de maneira sistêmica no organismo afetando, inclusive, a capacidade funcional do indivíduo.

Ozturk et al.22, observaram, em testes cardiopulmonar máximo, que em pacientes com SAHOS moderada e grave, quando comparados com indivíduos saudáveis, a capacidade de exercício foi prejudicada. Tal limitação ao exercício foi justificada por comprometimentos cardiovasculares. Já estudo realizado por Serafim et a ${ }^{23}$, que comparou indivíduos saudáveis e portadores de SAHOS, indicou que pacientes com a síndrome possuem uma capacidade de exercício reduzida, mesmo sem doenças cardiovasculares. A tolerância ao esforço foi avaliada através teste de esforço cardiopulmonar.

No presente estudo as comorbidades observadas foram hipertensão arterial sistêmica e dislipidemia, 45 dos 63 pacientes apresentaram pelo menos uma destas comorbidades, sendo que controladas por medicamentos. Embora fosse esperado que a tolerância ao esforço estivesse reduzida em pacientes com SAHOS, isso não foi observado neste estudo, reforçando a possibilidade da SAHOS por si só não afetar a tolerância ao esforço. Porém, testes que avaliam a capacidade funcional de maneira mais fidedigna são necessários para esclarecer a influência da SAHOS na tolerância ao esforço.

\section{CONCLUSÃO}

De acordo com os resultados do presente trabalho, podemos sugerir que a qualidade do sono não afeta a tolerância ao esforço dos indivíduos com SAHOS. No entanto, estudos com uma amostra maior, levando-se em consideração a gravidade da SAHOS e utilizando métodos mais acurados de avaliação da capacidade funcional devem ser realizados, a fim de que resultados mais abrangentes possam ser obtidos.

\section{AGRADECIMENTO}

Os autores agradecem o Laboratório do Sono e do Coração do Pronto Socorro Cardiológico de Pernambuco (PROCAPE).

Todos os autores declararam não haver qualquer potencial conflito de interesses referente a este artigo.

\section{REFERÊNCIAS}

1. Carskadon MA, Dement W. Normal human sleep: an overview. In: Kryger MH, Roth T, Dement WC, editors. Principles and practice of sleep medicine. $4^{\text {th }}$ ed. Philadelphia: W.B. Saunders 2005;13-23.

2. Buysse DJ, Germania A, Mould D, et al. Insomnia. In: BUYSSE DJ, ed. Sleep Disorders and psychiatry. Arlington, VA: American Psychiatric Publishing 2005;29-75

3. Roehrst T, Carskadon MA, Dement WC, et al. Daytime sleepiness and alertness. In: KRYGER MH, ROTHT, DEMENTWC, eds. Principles and practices of sleep medicine, 4th ed. Philadelphia: Elsevier Saunders 2005;39.

4. Chesson AL, Ferber RA, Fry JM, Grigg-Damberger M, Hartse KM, Hurwitz TD et al. Practice parameters for the indications for polysomnography and related procedures. American Sleep Disorders Association. Sleep 1997;22:406-20.

5. Antonelli IR, Marra C, Salvagni BL, Petrone A, Gemma A, Selvaggio D, et al. Does cognitive dysfunction conform to a distinctive pattern in obstructive sleep apnea syndrome? Journal Sleep Res 2004;13(1)79-86.

6. Martins RJ, Garcia AR, Garbin CAS, Sundefeld MLMM. Relação da classe econômica e qualidade do sono na ocorrência da disfunção temporomandibular. Rev. de Odontologia da Universidade Cidade de São Paulo 2007;75:269-19.

7. McNamara SG, Grunstein RR, Sullivan CE. Obstructive sleep apnea. Thorax 1993;48:754-64.

8. Sean MC, Apoor SG and Virend KS. Obstructive Sleep Apnea: physiology in medicine: a series of articles linking medicine with science 2005;142:187-197.

9. Young T, Peppard PE, Gottlieb DJ. Epidemiology of obstructive sleep apnea: a population health perspective. American Journal of Respiratory and Critical Care Medicine 2002;165:1217-39.

10. Roux F, D'Ambrosio C, Mohsenin V. Sleep-related breathing disorders and cardiovascular disease. American Journal of Medicine 2000; 108:396 -402.

11. Pedrosa RP, Lorenzi-Filho G, Drager LF. Síndrome da apneia obstrutiva do sono e doença cardiovascular. Rev Med (São Paulo) 2008;87(2):121-7.

12. American Thoracic Society. ATS Statement: Guidelines for the Six-Minute Walk Test 2002;166:111-117
13. American Academy of Sleep medicine Task Force. Sleep-related breathing disorders in adults: recommendations for syndrome definition and measurement techniques in clinical research. Sleep 1999;22-667-689.

14. Buysse DJ, Reynolds CF, Monk TH, Berman SR, Kupfer DJ. The Pittsburgh Sleep Quality Index: a new instrument for psychiatric practice and research. Res. Psychiatry 1989;193:213-28.

15. Bixler EO, Vgontzas AN, Lin H, Heve TT, Rein J, Vela-Bueno A, et al. Prevalence of sleep-disordered breathing in women - effects of gender. Am J Respir Crit Care Med. 2001;163(3 Pt 1):608-13.

16. Buysse DJ, Hall ML, Strollo PJ, Kamarck TW, Owens J, Lee L et al. Relationships between the Pittsburgh sleep quality index (PSQI), Epworth sleepiness scale (ESS), and clinical/polysomnography measures in a community sample. J Clin Sleep Med 2008;4(6):563-71.

17. Alameri $\mathrm{H}, \mathrm{Al}-\mathrm{KY}$, Bahammam A. Submaximal exercise in patients with severe obstructive sleep apnea. Sleep Breath 2010;145:151-14.

18. Rizzi CF, Cintra F, Risso T, Pulz C, TUFIK S, DE PAOLA A. et al. Exercise capacity and obstructive sleep apnea in lean subjects, Chest 2010;137(1):109-14

19. Plywaczewski R, Stocklosa A, Bielen P, Bednarek M, Czerniawska J, Jonczak L. et al. Test 6-minutowego chodu u chorych na obturacyjny bezdech senny. Pneumonol. Alergol. Pol. 2008;76:75-82.

20. Thomas EV, Barry AF, Kerstyn CZ, Sangal B, Adam TD, Agrawal V. Cardiorespiratory fitness and obstructive sleep apnea syndrome in morbidly obese patients. Sleep Medicine 2008;539:545-134.

21. Cintra F, Tufik S, De Paola A, Feres MC, Fujita LM, Oliveira W, Rizzi C, Poyares D. Perfil Cardiovascular em Pacientes com Apneia Obstrutiva do Sono. Arq Bras Cardiol. 2011;96(4):293-299.

22. Oztürk LM, Metin G, Cuhadaroglu A, TUTLUOGLU B. Cardiopulmonary responses to exercise in moderate-to-severe obstructive sleep apnea. Tuberk Toraks 2005;53(1):10-9.

23. Serafim NN, Dimitrios S, Smaragda K, Stavros D, Anatonia T, Athanasios T, et al. Early recovery period after exercise in obstructive sleep apnea syndrome. Clin. Cardiol. 2010;33(1):46-51. 